\title{
SPINAl CoRd InJUREd PATIENTS' KNOWLEDGE of PRESSURE SORES
}

\begin{abstract}
Despite much effort being put into educating spinal cord injured patients on pressure sore prevention, pressure sores remain a major cause of morbidity and mortality. This study sought to establish: i) paraplegics' knowledge on pressure sore prevention prior to discharge; ii) the influence of knowledge on pressure sore occurrence during hospitalization; iii) sources of paraplegics' knowledge on pressure sore prevention. A total of twenty-six men and two women with paraplegia aged between 18 and 45 years participated in the study. A prospective pre-test post-test method using a structured

MUDZI W, M.Sc'.

STEWART A, M.Sc Medicine"; EALES C, PhD²

${ }_{2}^{1}$ Ruwa Rehabilitation Hospital.

${ }^{2}$ Faculty of Health Sciences, Department of

Physiotherapy, University of the Witwatersrand. questionnaire was used to test paraplegics' knowledge on pressure sore prevention. The first interview was done within one week of mobilising in a wheelchair and the second one after two months or a few days before discharge. Pressure sore occurrence and the source of knowledge were also recorded. The results showed that paraplegics' knowledge on pressure sore prevention at discharge is incomplete. Knowledge gain does not seem to determine whether one will develop pressure sores or not. Physiotherapists (89\%), nurses (82\%) and occupational therapists (68\%) are the main educators of paraplegics on pressure sore prevention in rehabilitation hospitals.
\end{abstract}

\section{KEY WORDS: PARAPLEGIA, SPINAL CORD INJURY, PRESSURE SORES}

\section{A research project done at the University of the Witwatersrand in partial fulfillment of the MSc. degree in Physiotherapy.}

\section{INTRODUCTION}

Dinsdale (1974) puts the death toll among spinal cord injured patients due to pressure sores at between $7 \%$ and $8 \%$. Between $5 \%$ and $50 \%$ of spinal cord injured patients develop pressure sores during the period of acute care nursing and rehabilitation. Between $25 \%$ and $85 \%$ of all spinal cord injured patients develop pressure sores during their life time that are debilitating and life threatening (Dinsdale, 1974; Richardson and Meyer, 1981; Maklebust, 1999). Pressure sores remain a major cause of morbidity and mortality in spinal cord injured patients.

The expenses incurred in managing pressure sores in spinal cord injured patients during their lifetime range from a quarter of a million to two million American dollars (Lucke, 1999). Moody (1987) put the cost of pressure sores to the National Health Service in the United Kingdom at 200 million pounds per year. The costs associated with the hospital and health system in general include nurses' time, materials used, pressure relieving aids, drugs and extended stays in hospitals (De Vivo, 1997).
As mentioned by Manley (1978), information about the incidence of pressure sores and associated factors in South Africa is limited. His 1978 study done at Groote Schuur hospital in Cape Town put the cost of pressure sore treatment in excess of 2 million Rands per annum in the Western Cape alone.

Although pressure sores cause untold misery and suffering to the patient, delayed rehabilitation and a huge drain on the health service, they are more often than not, avoidable (Waterlow, 1988). Prevention, is agreed by many authors and clinicians, to be the treatment of choice. This must be done through patient education during the initial acute phase of hospitalisation. Any rehabilitation programme for spinal cord injured patients must include education for prevention of tissue breakdown.

The main aim in rehabilitation of the spinal cord injured patient is to attain the highest possible functional capacity within a short hospital stay. The whole rehabilitation process is aimed at preparing patients for self-care in the home setting. When teaching functional independence, pressure care education is an integral part of the programme. The main goal of patient education for spinal cord injured patients is to help patients implement positive choices required to prevent pressure sores (Garber et al., 1996; Sapountzi - Krepia, et al., 1998).

Patient education on pressure care normally includes teaching of risk factors such as immobility, positioning, medications, moisture, nutrition and hydration. In addition anatomical and physiological changes are explained. Most of the literature available focuses on mechanical and physiological causes of pressure sores. A few studies have looked at the influence of educational and behavioural interventions in helping prevent pressure sores (Anderson and Andberg, 1979; Rodriguez and Garber, 1994; Garber et al., 1996).

\section{CORRESPONDENCE TO:} W Mudzi Ruwa Rehabilitation Hospital P.O. Box 480 Ruwa, Zimbabwe

Tel: +26391260227 (cell) $+26373265 \mathrm{I} / 2839(\mathrm{w})$ 
Garber, et al (1996) examined pressure sore prevention and management techniques by spinal cord injured patients in the community. The results of their study showed that discharged spinal cord injured patients had incomplete information on prevention techniques prior to discharge into the community. They pointed out that the education of spinal cord injured patients on skin care is a hospital-based activity and that it is not known what information is retained or what behaviours are practiced after or at discharge.

Sumiya et al (1997) concluded that $72 \%$ (39 patients) of patients with paraplegia who had developed pressure sores developed them due to insufficient knowledge or improper techniques for prevention.

Rodriguez and Garber's (1994) study looked in part at the knowledge discharged spinal cord injured patients had on pressure sore prevention. Their study showed that what patients knew about pressure care was not what they did in practice. The knowledge that patients retained was incomplete and was not effectively translated into pressure sore prevention strategies. They also commented that it is not known how much of the information taught about pressure care is retained by patients at discharge.

There are no studies that have examined the level of knowledge patients have at discharge. No studies have evaluated the effectiveness of patient education programmes on pressure sore prevention during hospitalisation. Only a few studies have described the level of knowledge on pressure sore prevention of spinal cord injured patients in the community. (Garber et al., 1996; Rodriguez and Garber, 1994).

It is not known why presumably retained information is not translated into effective strategies for pressure sore management (Garber, et al., 1996). The need to objectively evaluate the effectiveness of educational programs during patients' hospitalisation cannot be overemphasised (Garber et al., 1996). We need to find out how much information is retained at discharge (Rodriguez and Garber, 1994).

A few studies have described who actually is involved in the clinical education of spinal cord injured patients. Basta (1991) looked at source(s) of knowledge of spinal cord injured patients for pressure sore prevention. This study revealed that nurses spent more time educating spinal cord injured patients than other professionals. The study noted that doctors, occupational therapists, physiotherapists and other clients were also instrumental in providing information on pressure sore prevention during rehabilitation of spinal cord injured patients.

\section{The purpose of this study was:}

1. To establish the knowledge paraplegics have on pressure care and sores during the hospitalisation period and at discharge.

2. To establish whether this knowledge has an influence on the occurrence of pressure sores during hospitalisation.

3. To establish the source(s) from which paraplegics get most of their knowledge regarding the prevention of pressure sores

\section{METHOD}

Ethical clearance was obtained from the Committee for Research on Human Subjects at the University of the Witwatersrand (protocol number: M000419). All subjects signed informed consent prior to their participation in the study. The purpose and procedure for the whole study was explained to the subjects.

The study included paraplegics admitted to two spinal injuries units in Johannesburg, one a government run hospital and one a private hospital. These patients came mainly from the greater Johannesburg area with all the hospitals being feeders to the two units.

The null hypotheses were stated as

(i) There is no significant difference in knowledge gain between paraplegics who develop pressure sores and those who do not.

(ii) The knowledge gain on pressure sore prevention in paraplegics does not influence the occurrence of pressure sores.

Patients were selected as they were admitted at the two hospitals. Patients were included in the study if they were paraplegic and aged between 18 and 45 years. They were not included in the study if they had any known chronic diseases e.g. diabetes, had a reduced level of consciousness or had any orthopaedic condition that made pressure relief difficult e.g. fractured arm.

The questionnaire sought to establish patients' knowledge on:

- Anatomical and physiological changes after injury

- Definition of pressure sore and risk factors

- Susceptibility of skin areas to pressures sores

- Prevention methods when using a wheelchair, in bed and other measures to prevent pressure sores

- Detection of early warning signs of skin breakdown

- Consequences of skin care neglect

- Source of knowledge on pressure care Data were collected using a pre-test post-test method. Two interviews were conducted with patients to test their knowledge on pressure care and sores using a structured questionnaire. The first interview was conducted during the first week of patients being mobilised in wheelchairs. The second interview was conducted within five days of discharge or when patients' length of stay was two months. Patients were asked to recall the information under given subheadings as shown above. No clues were given to aid with their recall. The questionnaire was administered on both occasions by the researcher.

Patients' responses were marked against a set of prepared correct responses. The responses were weighted to produce a possible correct total of 54 for the interview and converted into a percentage. The percentage then represented patients' knowledge on pressure care. The differences in performance between the first and second tests measured the knowledge gain. The occurrence of pressure sores was also recorded as well as patients' source of knowledge on pressure care. A patient was only recorded as having a pressure sore if they had a grade II stage wound or more. 
No changes were made to the way that patients were normally educated on pressure sore prevention.

Descriptive statistics were used to describe patients' knowledge on pressure sore prevention. Knowledge gain was analysed using the Rank Sum TwoSample (Mann-Whitney) test using the first and second interview results. The relationship between knowledge and occurrence of pressure sores was analysed using the students' two-sample ' $t$ '-test. The repeatability of the questionnaire was assessed using the method proposed by Bland and Altman (1986). The Repeatability Coefficient (RC) was calculated using a method adopted by the British Standards Institution.

Significance was set at the $0.05 \%$ confidence level for all tests.

The repeatability of the questionnaire was established by interviewing 20 patients twice on two consecutive days and established by the method proposed by Bland and Altman (1986). Using the method adopted by the British Standards Institution, the Reliability Coefficient (RC) for the questionnaire was 3 units. This means that we would expect two scores from two consecutive interviews within a short space of time e.g. day 1 and day 2, to be within 3 units of each other. This was considered tolerable and hence the questionnaire was repeatable.

\section{RESULTS}

The results presented are for a sample of 28 patients out of the original 30 . One was withdrawn after sustaining a fracture of the left arm. The other one was discharged prematurely (without prior notice) due to financial problems.

Minimum age was 19 , mean age 26.6 and maximum age 44 for the total sample $(\mathrm{N}=28)$. From the total sample $15(53,6 \%)$ were below the age of 30 . A total of 11 subjects $(39 \%)$ developed pressure sores during the research period.

For the pressure sore group, the minimum knowledge gain was $6.1 \%$ while the maximum knowledge gain was $184.6 \%$. For the no pressure sore group, the minimum percentage gain was $-26.8 \%$ while maximum percentage gain was $1550 \%$. A maximum gain of $1550 \%$ suggested the presence of outliers. The outliers were four people who started with a score in Test $1 \leq 7 \%$ in the no pressure sore group. Their major gain caused a skewed distribution and hence there was need for an adjustment (by removal of outliers) when doing further calculations on the data.

Of the total patient sample 24 (86\%) mentioned body changes related to altered sensation, paralysis and reduced mobility but only 1 (4\%) mentioned altered blood circulation as a change. More than $95 \%$ of the total patients recalled that pressure sores are caused by unrelieved pressure in either lying or sitting.

The pelvic areas (sacrum 93\%, ischium $86 \%$ and greater trochanter $82 \%$ ) as being prone to pressure sores were mentioned most. Other areas that received the most mention were heels $(61 \%)$, elbows $(61 \%)$ and the medial and lateral knee condyles $(54 \%)$.

Pressure relief was the most known method of prevention of pressure sores when using a wheelchair. Only $8(29 \%)$ patients mentioned the use of cushions as a means of preventing pressure sores.

Table 1: Rank Sum Two-Sample (Mann-Whitney) Test for Knowledge Gain for the Pressure Sore and No Pressure Sore Groups after adjusting for the outliers.

\begin{tabular}{|l|c|c|c|c|}
\hline $\begin{array}{l}\text { Pressure } \\
\text { Sore Groups }\end{array}$ & Rank Sum & $\begin{array}{c}\text { Sample Size } \\
(\mathrm{N})\end{array}$ & U Statistics & Mean Rank \\
\hline Pressure Sores & 113 & 11 & 47 & 10.3 \\
\hline No Pressure Sores & 187 & 13 & 96 & 14.4 \\
\hline Total & 300 & 24 & & 0.16 \\
\hline \multicolumn{5}{|l|}{ Two-Tailed P-value for normal approximation } \\
\hline \multicolumn{5}{|l|}{ Cases included } \\
\hline
\end{tabular}

The $p$ value of 0.16 indicates that the null hypotheses can be accepted. This stated that there was no significant difference in the knowledge gain between those who developed pressure sores and those who did not.

Table 2: Students' Two-Sample ' $t$ ' test results for influence of knowledge gained on occurrence of pressure sores for the total group after adjusting for the outliers.

\begin{tabular}{|c|c|c|c|c|}
\hline $\begin{array}{l}\text { Pressure } \\
\text { Sore Groups }\end{array}$ & Mean & $\begin{array}{c}\text { Sample Size } \\
\text { (N) }\end{array}$ & $\begin{array}{l}\text { Standard } \\
\text { Deviation }\end{array}$ & $\begin{array}{c}\text { Standard } \\
\text { Error }\end{array}$ \\
\hline Pressure Sores & 49.95 & 11 & 52.76 & 15.91 \\
\hline No Pressure Sores & 73.03 & 13 & 57.09 & 15.83 \\
\hline
\end{tabular}

The $p$ value of 0.32 indicates that the null hypotheses can be accepted. This stated that there was no significant difference in the influence of knowledge gain on the occurrence of pressure sores.

The physiotherapist ( $98 \%$ ) was the most involved professional in pressure sore prevention education followed by the nurse ( $82 \%$ ) and occupational therapist $(68 \%)$ respectively. Only one individual reported having used posters as a source of information. 


\section{DISCUSSION}

The results clearly show that the information paraplegics had on pressure sore prevention was incomplete. Although there was evident gain in knowledge, the amount of information that paraplegics could recall at discharge regarding pressure sore prevention was not adequate.

This agrees with the findings by Rodriguez and Garber (1994) and Garber, et al. (1996) whose studies looked at discharged spinal cord injury patients. The reason why their subjects could not recall adequate information on pressure sore prevention methods was, as they suggested, most probably because patients were discharged into the community with less information. This appears to have been a reasonable conclusion because as the results for this research show, paraplegics who were just about to be discharged from the two hospitals into the community did not have complete information on pressure sore prevention.

Rodriguez and Garber (1994) found that weight shift or turning when in bed were the most often-practiced pressure sore prevention techniques. This finding is supported by the fact that turning in bed and pressure relief when in the wheelchair were the most recalled methods of pressure sore prevention in this study. However unlike their study where the use of cushions was ranked as the most important method of pressure sore prevention it only received a mention from $29 \%$ of the total sample for this study. This might be because cushions were not emphasised as being important during pressure sore prevention education. As a result of budgetary constraints some paraplegics are being mobilised without cushions at the rehabilitation hospitals and this might account for the perceived lack of importance of cushions.

Anderson and Andberg (1979) showed the psychosocial well being of patients has an effect on the ability of patients to undergo any form of education. This area needs to be investigated because many paraplegic patients spend a lot of time in the denial stage, still coping with their sudden disability. Learning such vital techniques is likely to be the last thing they are ready for at this stage.
Some patients' length of stay is determined by fiscal rather than medical considerations. This does not allow patients to gain adequate knowledge on pressure sore prevention, as patients are increasingly being discharged after short hospital stays. Early, effective education on pressure sore prevention is essential.

Garber, et al. (1996) concluded that little emphasis was being given to management action once a pressure sore has developed. This appears also to have been the case in this study where the majority of patients $(75 \%)$ thought the best thing was just to notify the health personnel about the pressure sore. Only $43 \%$ of the total sample said they would have to remove pressure from the affected area. There is a need to emphasise the fact that prevention and successful management of pressure sores is the individual's responsibility and not that of the hospital.

The finding that knowledge gain does not have a significant influence on whether one develops pressure sores or not suggests a discrepancy between what is done in practice and what paraplegics know about pressure sore prevention. This agrees with Rodriguez and Garber (1994) 's findings. This is however difficult to quantify because one needs to first look at the degree of adherence to the prevention programmes among spinal cord patients.

As shown by Sumiya et al (1997), an improvement in self-care among paraplegics helps in preventing pressure sores. The ability of patients to gain adequate information on pressure sore prevention during rehabilitation is threatened by reduced length of stay and the present emphasis that is being placed on financial instead of medical sense.

This study showed that physiotherapists are the most involved professionals in the education of spinal cord injured patients in a rehabilitation setting. The nurses and occupational therapists follow them respectively. Caution however needs to be exercised when interpreting these results. Interviews were done by a physiotherapist who was not working at either of the two rehabilitation hospitals involved in the study. However, the fact that patients knew from the introductions done during the first interview might have swayed the results in favour of physiotherapists (without taking anything away from them though).

Previous studies (Basta, 1991) have shown nurses as being more engaged in pressure sore education than other professions followed by therapists, a finding that is not very different from this study. The reason why therapists might have scored such a high popularity as pressure sore educators is that they spend more time with patients than any other professional at this stage of rehabilitation. The same reason can also be used to explain nurses' involvement as pressure sore educators.

\section{Limitations of the Study:}

1. The target population (spinal cord injury patients) is much bigger and more diverse than the studied sample and hence caution needs to be exercised when extrapolating these results to the entire population.

2. Although having knowledge is a pre-requisite for a pressure sore free life, there are other factors that contribute to pressure sore formation, which cannot be controlled.

\section{CONCLUSION AND RECOMMENDATIONS}

Spinal cord injured patients' knowledge on pressure sore prevention (at the two hospitals studied) at discharge appears to be incomplete. They are being discharged into the community with inadequate information on pressures sore prevention, which is a cause for concern about their ability to cope when at home.

Knowledge gain does not seem to determine whether patients will get pressure sores or not. This shows a discrepancy between what paraplegics recall on pressure sore prevention and what they actually do in practice.

Physiotherapists (98\%), nurses (82\%) and occupational therapists $(68 \%)$, in that order, are the leading sources of pressure care knowledge for spinal cord injured patients in the two hospitals studied.

\section{Clinical Recommendations:}

1. There is a need to evaluate paraplegics' knowledge at least three weeks before discharge to establish 
their weak areas of knowledge and improve them before discharge.

2. Patient education should be spread out throughout their length of stay (entire rehabilitation period) and not just confined to the first few weeks.

3. There is a need to involve patients' families and friends in education programmes so that they can help to reinforce their knowledge especially when patients are discharged thus allowing for continuity.

4. Resources allowing, patients would benefit more from follow-up after discharge or workshops where they are called back for regular updating on pressure sore prevention.

\section{REFERENCES}

Anderson T P, Andberg M M 1979 Psychosocial Factors Associated with Pressure Sores. Archives of Physical Medicine and Rehabilitation 60: 341 - 346
Basta M S 1991 Pressure Sore Prevention Education with the Spinal Cord Injured. Rehabilitation Nursing 16(1): 6 - 8

Bland M J, Altman D G 1986 Statistical Methods for Assessing Agreement Between Two Methods of Clinical Measurement. The Lancet: $307-310$

DeVivo M J 1997 Causes and costs of spinal cord injury in the United States of America. Spinal cord 35: $809-813$

Dinsdale S M 1974 Decubitus Ulcers: Role of Pressure and Friction in Causation. Archives of Physical Medicine and Rehabilitation 55: $147-152$

Garber S L, Rintala D H, Rossi D C, Hart K A, Fuhrer M J 1996 Reported Pressure Ulcer Prevention and Management Techniques by Persons with Spinal Cord Injury. Archives of Physical Medicine and Rehabilitation 77: 744 - 749

Lucke K T 1999 Outcomes of Nurse Caring as Perceived by Individuals with Spinal Cord [njury During Rehabilitation. Rehabilitation Nursing 24(6): $247-253$

Maklebust J 1999 Interrupting the Pressure Ulcer Cycle. Nursing Clinics of North America 34 (4): 861 - 871
Manley M T 1978 Incidence, Contributory Factors and Costs of Pressure Sores. South African Medical Journal 53: 217 - 222

Moody 1987 Relieving the Pressure. Nursing Times 83(39): 53

Richardson R R, Meyer P R Jr 1981 Prevalence and Incidence of Pressure Sores in Acute Spinal Cord Injuries. Paraplegia 19(4): $235-247$

Rodriguez G P, Garber S L 1994. Prospective study of pressure ulcer risk in spinal cord injury patients. Paraplegia 32: 150 - 158

Sapountzi-Krepia D, Soumilas A, Papadakis N, Sapkas G, Nomicos J, Theodossopoulou E, Dimitriadou A 1998 Post Traumatic Paraplegics Living in Athens: The Impact of Pressures Sores and Urinary Tract Infections on Everyday Life Activities. Spinal Cord 36: $432-437$

Sumiya T, Kawamura $\mathrm{K}$, Tokuhiro A, Takechi H, Ogata H 1997 A survey of wheelchair use by paraplegic individuals in Japan. Part 2: Prevalence of Pressure Sores. Spinal Cord 35: 595 -598

Waterlow J 1988 Prevention is Cheaper than Cure. Nursing Times 84 (25): 69 - 70 